\title{
Gemma Robles
}

José M. Méndez

\section{CONVERSE ACKERMANN PROPERTY AND CONSTRUCTIVE NEGATION DEFINED WITH A NEGATION CONNECTIVE*}

\begin{abstract}
The Converse Ackermann Property is the unprovability of formulas of the form $(A \rightarrow B) \rightarrow C$ when $C$ does contain neither $\rightarrow$ nor $\neg$. Intuitively, the CAP amounts to rule out the derivability of pure non-necessitive propositions from non-necessitive ones. A constructive negation of the sort historically defined by, e.g., Johansson is added to positive logics with the CAP in the spectrum delimited by Ticket Entailment and Dummett's logic LC.
\end{abstract}

Keywords: Converse Ackermann Property, Constructive negation, Relational ternary semantics, Relevance logics.

\section{Introduction}

Consider a propositional logic $L$ with the customary relevance logics negation axioms. Generally speaking, the logic $L$ has the Ackermann Property (AP)

\footnotetext{
*An abstract of this paper was read at the Logic Colloquium '04 (ASL European Summer Meeting), Turin, Italy, 24-31, July, 2004.

Work partially supported by the research project HUM2005-05707 of the Spanish Ministry of Education and Science.

We thank a referee of LLP for his (her) comments that improved a previous version of this paper.
} 
if any scheme of the form $A \rightarrow(B \rightarrow C)$ is unprovable when $\rightarrow$ does not appear in $A$, that is, when $A$ does not contain at least an implicative formula. (But the meaning of the property can be sharpened. See [1], §22.1.)

Intuitively, and roughly speaking, the AP amounts to rule out the derivability of necessitive propositions from pure non-necessitive ones, a necessitive proposition being one of the form $\square B$ (see again [1], §22.1). In other words, the AP would mean that "purely truth functional propositions never entail entailments" ([1] p. 237).

Conversely, $L$ has the Converse Ackermann Property (CAP) if $(A \rightarrow$ $B) \rightarrow C$ is unprovable when $\rightarrow$ does not appear in $C$, i.e., when $C$ does not contain at least one implicative formula. Intuitively and generally speaking, if the CAP is predicable of $L$, necessitive propositions would never entail pure non-necessitive ones; entailments would never entail purely truth functional propositions.

Consider now a constructive negation in the historical sense (that defined by Kolmogorov [5] and Johansson [3], for example). It is known that this type of negation can be introduced either by a falsity constant $\perp$ or else by a unary connective $\neg$. If it is introduced by means of the constant, we build up a definitional extension of positive intuitionistic logic: we add the constant to the positive logic language without adding any specific axiom about $\perp$ and define $\neg A==_{\text {df }} A \rightarrow \perp$. Then, it is the positive logic which, so to speak, reveals "its own underlying concept of negation".

If minimal negation is introduced by the unary connective, as it is known, it is formulated by adding to positive intuitionistic logic the axioms

$$
\begin{array}{lr}
\alpha 1 . A \rightarrow \neg \neg A & \text { weak double negation } \\
\alpha 2 .(A \rightarrow B) \rightarrow(\neg B \rightarrow \neg A) & \text { weak contraposition } \\
\alpha 3 .(A \rightarrow B) \rightarrow[(A \rightarrow \neg B) \rightarrow \neg A)] & \text { weak reductio }
\end{array}
$$
or any other set of equivalent axioms.

Let us name $\mathrm{I}_{\mathrm{m}}$ the logic defined by means of $\perp$ and $\mathrm{I}_{\mathrm{m}^{\prime}}$ the logic defined by means of $\neg$. It is a well known fact that $I_{m}$ and $I_{m^{\prime}}$ are deductively equivalent.

Now, it is clear that a "minimal negation" can always be introduced by means of a falsity constant in any positive logic. However, it is not always so if it is introduced by means of the unary connective in weaker positive logics (but we cannot develop this point here). Nevertheless, it is obvious that the stronger the positive logic is, the stronger its underlying minimal negation will be. 
Let us now label a "Johansson negation" (NJ) that type of negation resulting from adding $\alpha 1, \alpha 2$ and $\alpha 3$ to any positive logic. Well, a NJ is the "constructive part" of the De Morgan negation characteristic of standard relevance logics, which is, as it is konwn, the result of adding to the positive logics the axioms

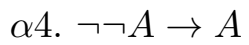

$$
\begin{aligned}
& \alpha 5 .(A \rightarrow \neg B) \rightarrow(B \rightarrow \neg A) \\
& \alpha 6 .(\neg A \rightarrow B) \rightarrow[(\neg A \rightarrow \neg B) \rightarrow A]
\end{aligned}
$$

strong double negation weak contraposition strong reductio

or any other set of equivalent axioms.

In consequence, a Johansson negation is interesting in itself and so it is independently of minimal intuitionism.

As it was remarked above, in weaker positive logics minimal negation is correspondingly weaker (weaker, anyway, than intuitionistic minimal negation). Is it impossible, however, to introduce a NJ in these logics?

The aim of this paper is to show how to introduce this type of negation by means of a unary connective in logics with the CAP, so let us return to the logics with this property. If negation is understood along the lines discussed above, it is clear that the AP and the CAP have to be redefined.

Definition $1(\mathrm{AP} \neg)$. A propositional logic $L$ has the AP if $A \rightarrow(B \rightarrow C)$ is unprovable when $A$ contains neither $\rightarrow$ nor $\neg$.

Definition $2(\mathrm{CAP} \neg)$. A propositional logic $L$ has the CAP if $(A \rightarrow B) \rightarrow$ $C$ is unprovable when $C$ contains neither $\rightarrow$ nor $\neg$.

Generally (and syntactically) speaking, logics with the CAP are characterized by the absence of assertion, i.e.,

$$
A \rightarrow[(A \rightarrow B) \rightarrow B]
$$

and contraction

$$
[A \rightarrow(A \rightarrow B)] \rightarrow(A \rightarrow B)
$$

though restricted versions of these theses are present. In particular, both of them are valid when $B$ is an implicative formula. So, logics with the CAP are the natural bridge between contractionless logics (see the foundational Ono and Komori [9] or the overview Kowalski and Ono [6]) and logics with contraction. 
Next, we briefly summarize the results related to the Converse Ackermann Property. The problem concerning which systems do have the CAP is first posed in [1], §8.12 and in [7] it is solved for positive logics. Regarding negation, in [8], a so-called "semiclassical" negation and in [4], a strong negation are added to the positive logics in [7]. Finally, in [11] and [12] an intuitionistic negation is added to Intuitionistic and Dummett's LC positive logics with the CAP. The aim of this paper is to show how to introduce a constructive negation of the kind just discussed in any logic including $\mathrm{T}_{+}^{o}$, i.e., Positive Ticket Entailment with the CAP. In addition, we shall introduce this constructive negation in some prominent logics in the spectrum between $\mathrm{T}_{\mathrm{c}}^{\mathrm{o}}$ and $\mathrm{LC}_{\mathrm{c}}^{\mathrm{o}}$ (see below, structure of the paper).

Unlike the negation of intuitionistic (and minimal) logic, this notion of negation is devoid of paradoxical theses as $A \rightarrow(\neg A \rightarrow B)$ and others of the sort and it could intuitively be described as containing the following essential principles: contraposition (A2, T2 below), double negation (T1), reductio (T15, T16, T17), interdefinition between $\rightarrow$ and $\wedge$ (T18, T19, T20, T21), De Morgan laws (T9, T10) and non-contradiction (T22).

Before describing the structure of the paper, we recall the abbreviations for some well known propositional logics which we will have to mention. T, E, R, RMO, S4, I and LC refer to Ticket Entailment, Entailment logic, Relevance logic, Relevance logic with mingle, Lewis's Modal logic S4, Intuitionistic logic and Dummett's superintuitionistic logic LC, respectively $\left(\mathrm{T}_{+}\right.$, $\mathrm{E}_{4}$ etc. refer to the positive fragments).

Now, the structure of the paper is as follows. In $\S 2$, the logic $T_{c}^{o}$ is syntactically defined. $\mathrm{T}_{\mathrm{c}}^{\mathrm{o}}$ is $\mathrm{T}$ with the $\mathrm{CAP}$ and constructive negation. In $\S 3$, $4, \mathrm{~T}_{\mathrm{c}}^{\mathrm{o}}$ semantics are defined and semantic consistency and completeness are proved. In addition, a variety of syntactical and semantic axiomatizations of $\mathrm{T}_{+}^{o}$ are provided. In $\S 5$, we recall the restrictions with the $\mathrm{CAP}$ of the unrestricted posive logics mentioned above (that is, the logics in [7] except for $\mathrm{LC}_{+}^{o}$, which was defined in [12]), and in $\S 6$, we add the constructive negation to each one of these logics. Finally, in $\S 7$, it is proved that the CAP is a property of the logics defined. We assume reader's acquaintance with the relational ternary semantics and especially, with the logic $\mathrm{TW}_{+}$, that is, Ticket Entailment without the contraction axiom.

\section{The $\operatorname{logic} \mathbf{T}_{\mathbf{c}}^{\mathrm{o}}$}

The logic $\mathrm{TW}_{+}$(positive Ticket Entailment without contraction) is axiomatized as follows (see, e.g., [1]) 
Axioms:

a1. $A \rightarrow A$

a2. $(B \rightarrow C) \rightarrow[(A \rightarrow B) \rightarrow(A \rightarrow C)]$

a3. $(A \rightarrow B) \rightarrow[(B \rightarrow C) \rightarrow(A \rightarrow C)]$

a4. $(A \wedge B) \rightarrow A \quad / \quad(A \wedge B) \rightarrow B$

a5. $[(A \rightarrow B) \wedge(A \rightarrow C)] \rightarrow[A \rightarrow(B \wedge C)]$

a6. $A \rightarrow(A \vee B) \quad / \quad B \rightarrow(A \vee B)$

a7. $[(A \rightarrow C) \wedge(B \rightarrow C)] \rightarrow[(A \vee B) \rightarrow C)]$

a8. $[A \wedge(B \vee C)] \rightarrow[(A \wedge B) \vee(A \wedge C)]$

Rules of inference:

Modus ponens: if $\vdash A$ and $\vdash A \rightarrow B$, then $\vdash B$

Adjunction: if $\vdash A$ and $\vdash B$, then $\vdash A \wedge B$

The logic $\mathrm{T}_{+}^{o}$ is positive Ticket Entailment (see [1]) with the CAP (see [7]). It is the result of adding the restricted contraction axiom

A1. $[A \rightarrow[A \rightarrow(B \rightarrow C)]] \rightarrow[A \rightarrow(B \rightarrow C)]$

to $\mathrm{TW}_{+}$. The following theorems belong to $\mathrm{T}_{+}^{\mathrm{o}}$ :

t1. $(A \rightarrow B) \rightarrow[[A \rightarrow[B \rightarrow(C \rightarrow D)]] \rightarrow[A \rightarrow(C \rightarrow D)]]$

t2. $[A \rightarrow[B \rightarrow(C \rightarrow D)]] \rightarrow[(A \rightarrow B) \rightarrow[A \rightarrow(C \rightarrow D)]]$

t3. $[A \rightarrow[B \rightarrow(C \rightarrow D)]] \rightarrow[(A \wedge B) \rightarrow(C \rightarrow D)]$

The logic $\mathrm{T}_{\mathrm{c}}^{\mathrm{o}}$ is $\mathrm{T}_{+}^{o}$ with the constructive negation we have discussed. It is defined by adding the unary connective $\neg$ (negation) to the positive language together with the axioms:

A2. $(A \rightarrow \neg B) \rightarrow(B \rightarrow \neg A)$

A3. $B \rightarrow[(A \rightarrow \neg B) \rightarrow \neg A]$

The axiom A2 is (a form of) weak contraposition (the other is T2 below) and A3 is permuted A2. The following theses are derivable in $\mathrm{T}_{\mathrm{c}}^{\mathrm{o}}$ (a sketch of the proof is given on the right-hand side of each theorem):

T1. $A \rightarrow \neg \neg A$

T2. $(A \rightarrow B) \rightarrow(\neg B \rightarrow \neg A)$ 
T3. $\neg B \rightarrow[(A \rightarrow B) \rightarrow \neg A]$ A3, T1

T4. $A \rightarrow[(A \rightarrow \neg B) \rightarrow \neg B]$ A2, A3

T5. $[A \rightarrow(B \rightarrow \neg C)] \rightarrow[B \rightarrow(A \rightarrow \neg C)]$ $\mathrm{T} 4$

T6. $B \rightarrow[[A \rightarrow(B \rightarrow \neg C)] \rightarrow(A \rightarrow \neg C)]$ $\mathrm{T} 4$

T7. $\neg A \rightarrow[A \rightarrow \neg(A \rightarrow A)]$ A2, T3

T8. $[A \rightarrow \neg(A \rightarrow A)] \rightarrow \neg A$ A3

T9. $\neg(A \vee B) \leftrightarrow(\neg A \wedge \neg B)$ A2, T2

T10. $(\neg A \vee \neg B) \rightarrow \neg(A \wedge B)$

Now, by using A1 in the form

$$
[A \rightarrow[A \rightarrow(B \rightarrow \neg C)]] \rightarrow[A \rightarrow(B \rightarrow \neg C)]
$$

$\mathrm{T} 7$ and $\mathrm{T} 8$, we prove

T11. $[A \rightarrow(A \rightarrow \neg B)] \rightarrow(A \rightarrow \neg B)$ A1, T7, T8

T12. $[A \rightarrow(B \rightarrow \neg C)] \rightarrow[(A \rightarrow B) \rightarrow(A \rightarrow \neg C)]$

$\mathrm{T} 11$

T13. $(A \rightarrow B) \rightarrow[[A \rightarrow(B \rightarrow \neg C)] \rightarrow(A \rightarrow \neg C)]$ T11

T14. $[A \rightarrow(B \rightarrow \neg C)] \rightarrow[(A \wedge B) \rightarrow \neg C]$ $\mathrm{T} 13$

T15. $(A \rightarrow \neg A) \rightarrow \neg A$ A2, A3, T11

T16. $(A \rightarrow B) \rightarrow[(A \rightarrow \neg B) \rightarrow \neg A]$ A2, T15

T17. $(A \rightarrow \neg B) \rightarrow[(A \rightarrow B) \rightarrow \neg A]$ T5, T16

T18. $(A \wedge B) \rightarrow \neg(A \rightarrow \neg B)$ A2, T4, T14

T19. $(A \rightarrow \neg B) \rightarrow \neg(A \wedge B)$ A2, T18

T20. $(A \rightarrow B) \rightarrow \neg(A \wedge \neg B)$ T1, T19

T21. $(A \wedge \neg B) \rightarrow \neg(A \rightarrow B)$

T22. $\neg(A \wedge \neg A)$

Note that given $\mathrm{T}_{+}^{\mathrm{o}}$ and weak contraposition (A2, A3), the reductio theorems T11-T22 are derivable. In other words, any logic including $\mathrm{T}_{+}^{o}$ and with weak contraposition necessarily has the reductio axioms T11-T22 (on the aplication of the label "reductio axioms", see [10]). On the other hand, notice that $\mathrm{T} 4, \mathrm{~T} 5, \mathrm{~T} 6, \mathrm{~T} 11, \mathrm{~T} 12, \mathrm{~T} 13$ and $\mathrm{T} 14$ are restricted versions of assertion

$$
A \rightarrow[(A \rightarrow C) \rightarrow C]
$$


permutation

$$
[A \rightarrow(B \rightarrow C)] \rightarrow[B \rightarrow(A \rightarrow C)]
$$

conditioned modus ponens

$$
B \rightarrow[[A \rightarrow(B \rightarrow C)] \rightarrow(A \rightarrow C)]
$$

contraction

$$
[A \rightarrow(A \rightarrow C)] \rightarrow(A \rightarrow C)
$$

autodistribution of the conditional

$$
[A \rightarrow(B \rightarrow C)] \rightarrow[(A \rightarrow B) \rightarrow(A \rightarrow C)]
$$

permuted autodistribution of the conditional

$$
(A \rightarrow B) \rightarrow[[A \rightarrow(B \rightarrow C)] \rightarrow(A \rightarrow C)]
$$

and the importation axiom

$$
[A \rightarrow(B \rightarrow C)] \rightarrow[(A \wedge B) \rightarrow C]
$$

to the case in which $C$ is a negative formula. We remark that these unrestricted versions are unprovable in all the logics with CAP discussed in this paper. This fact easily follows from the set of matrices in $\S 7$. Finally, we remark that T4-T6 and T11-T14 can be generalized:

T4g. $A \rightarrow\left\{\left[A \rightarrow\left[B_{1} \rightarrow\left(\left(\ldots\left(B_{n} \rightarrow \neg C\right) \ldots\right)\right)\right]\right] \rightarrow\left[B_{1} \rightarrow\right.\right.$

$$
\left.\left.\left(\left(\ldots\left(B_{n} \rightarrow \neg C\right) \ldots\right)\right)\right]\right\}
$$

T5g. $\quad\left\{A \rightarrow\left[B_{1} \rightarrow\left(\left(\ldots \rightarrow\left(B_{n} \rightarrow \neg C\right) \ldots\right)\right)\right]\right\} \rightarrow\left\{B_{1} \rightarrow\left[A \rightarrow\left(B_{2} \rightarrow\right.\right.\right.$

$$
\left.\left.\left(\left(\ldots\left(B_{n} \rightarrow \neg C\right) \ldots\right)\right)\right]\right\}
$$

T6g. $B_{1} \rightarrow\left\{\left[A \rightarrow\left[B_{1} \rightarrow\left(\left(\ldots\left(B_{n} \rightarrow \neg C\right) \ldots\right)\right)\right]\right] \rightarrow\left[A \rightarrow\left[B_{2} \rightarrow\right.\right.\right.$

$$
\left.\left.\left.\left(\left(\ldots\left(B_{n} \rightarrow \neg C\right) \ldots\right)\right)\right]\right]\right\}
$$

T11g. $\left\{A \rightarrow\left[A \rightarrow\left[B_{1} \rightarrow\left(\left(\ldots\left(B_{n} \rightarrow \neg C\right) \ldots\right)\right)\right]\right]\right\} \rightarrow\left\{A \rightarrow\left[B_{1} \rightarrow\right.\right.$

$$
\left.\left.\left(\left(\ldots\left(B_{n} \rightarrow \neg C\right) \ldots\right)\right)\right]\right\}
$$

T12g. $\left\{A \rightarrow\left[B_{1} \rightarrow\left(\left(\ldots\left(B_{n} \rightarrow \neg C\right) \ldots\right)\right)\right]\right\} \rightarrow\left\{\left(A \rightarrow B_{1}\right) \rightarrow[A \rightarrow\right.$

$$
\left.\left.\left[B_{2} \rightarrow\left(\left(\ldots\left(B_{n} \rightarrow \neg C\right) \ldots\right)\right)\right]\right]\right\}
$$

T13g. $\left(A \rightarrow B_{1}\right) \rightarrow\left\{\left[A \rightarrow\left[B_{1} \rightarrow\left(\left(\ldots\left(B_{n} \rightarrow \neg C\right) \ldots\right)\right)\right]\right] \rightarrow[A \rightarrow\right.$

$$
\left.\left.\left[B_{2} \rightarrow\left(\left(\ldots\left(B_{n} \rightarrow \neg C\right) \ldots\right)\right)\right]\right]\right\}
$$


T14g. $\left\{A \rightarrow\left[B_{1} \rightarrow\left(\left(\ldots \rightarrow\left(B_{n} \rightarrow \neg C\right) \ldots\right)\right)\right]\right\} \rightarrow\left\{\left(A \wedge B_{1}\right) \rightarrow\right.$

$$
\left.\left[B_{2} \rightarrow\left(\left(\ldots\left(B_{n} \rightarrow \neg C\right) \ldots\right)\right)\right]\right\}
$$

These generalized theorems are proved as follows:

$$
\begin{aligned}
& \mathrm{T} 4 \mathrm{~g}(n=i): \mathrm{T} 4 \mathrm{~g}(n=i-1), \mathrm{T} 5 \mathrm{~g}(n=i) \\
& \mathrm{T} 5 \mathrm{~g}(n=i): \mathrm{T} 4 \mathrm{~g}(n=i-1) \\
& \operatorname{T} 6 \mathrm{~g}(n=i): \operatorname{T} 4 \mathrm{~g}(n=i-1) \\
& \operatorname{T11g}(n=i): \operatorname{T5g}(n=i), \operatorname{T5g}(n=i+1), \operatorname{T11g}(n=i-1) \\
& \text { T12g }(n=i): \text { T5g }(n=i+1), \operatorname{T11g}(n=i-1) \\
& \operatorname{T} 13 \mathrm{~g}(n=i): \operatorname{T} 11 \mathrm{~g}(n=i-1) \\
& \operatorname{T} 14 \mathrm{~g}(n=i): \operatorname{T} 13 \mathrm{~g}(n=i)
\end{aligned}
$$

For instance, let us prove $\operatorname{T} 11 \mathrm{~g}(n=k)$.

Proof. By T11g $(n=k-1)$ :

$$
\begin{aligned}
(1) . & \left\{A \rightarrow\left[A \rightarrow\left[B_{2} \rightarrow\left(\left(\ldots\left(B_{k} \rightarrow \neg C\right) \ldots\right)\right)\right]\right]\right\} \rightarrow\left\{A \rightarrow \left[B_{2} \rightarrow\right.\right. \\
& \left.\left.\left(\left(\ldots\left(B_{k} \rightarrow \neg C\right) \ldots\right)\right)\right]\right\}
\end{aligned}
$$

By the prefixing rule of $\mathrm{TW}_{+}$

$$
\begin{aligned}
(2) . & \left\{B_{1} \rightarrow\left[A \rightarrow\left[A \rightarrow\left[B_{2} \rightarrow\left(\left(\ldots\left(B_{k} \rightarrow \neg C\right) \ldots\right)\right)\right]\right]\right]\right\} \rightarrow\left\{B_{1} \rightarrow\right. \\
& {\left.\left[A \rightarrow\left[B_{2} \rightarrow\left(\left(\ldots\left(B_{k} \rightarrow \neg C\right) \ldots\right)\right)\right]\right]\right\} }
\end{aligned}
$$

By applying $\operatorname{T} 5 \mathrm{~g}(n=k)$ in the consequent

$$
\text { (3). } \begin{aligned}
& \left\{B_{1} \rightarrow\left[A \rightarrow\left[A \rightarrow\left[B_{2} \rightarrow\left(\left(\ldots\left(B_{k} \rightarrow \neg C\right) \ldots\right)\right)\right]\right]\right]\right\} \rightarrow\{A \rightarrow \\
& {\left.\left[B_{1} \rightarrow\left[B_{2} \rightarrow\left(\left(\ldots\left(B_{k} \rightarrow \neg C\right) \ldots\right)\right)\right]\right]\right\} }
\end{aligned}
$$

By applying $\operatorname{T} 5 \mathrm{~g}(n=k), \operatorname{T} 5 \mathrm{~g}(n=k+1)$ in the antecedent

(4). $\left\{A \rightarrow\left[A \rightarrow\left[B_{1} \rightarrow\left[B_{2} \rightarrow\left(\left(\ldots\left(B_{k} \rightarrow \neg C\right) \ldots\right)\right)\right]\right]\right]\right\} \rightarrow\{[A \rightarrow$

$$
\left.\left[B_{1} \rightarrow\left[B_{2} \rightarrow\left(\left(\ldots\left(B_{k} \rightarrow \neg C\right) \ldots\right)\right)\right]\right]\right\}
$$

That is, $\operatorname{T} 11 \operatorname{g}(n=k)$.

On the relationships between A2, A3 and T1-T8, we have (the proof is left to the reader):

Proposition 1. i. Given $\mathrm{TW}_{+}, \mathrm{A} 2$ is interchangeable with $\mathrm{T} 4$ or $\mathrm{T} 5$.

ii. Given $\mathrm{TW}_{+}$and $\mathrm{A} 3, \mathrm{~T} 2$ and $\mathrm{T} 6$ are equivalent to $\mathrm{A} 2$.

iii. Given $\mathrm{TW}_{+}$and A2, A3 is equivalent to $\mathrm{T} 3$.

iv. Given $\mathrm{TW}_{+}$and $\mathrm{A} 2, \mathrm{~A} 3$ is equivalent to $\mathrm{T} 1$ and $\mathrm{T} 2$. 
And regarding the generalized versions, it is proved

Proposition 2. The following group of theses do axiomatize $T_{c}^{o}$ :

i. $\mathrm{A} 3, \mathrm{~T} 7$ and $\mathrm{T} 4 \mathrm{~g}$ (or $\mathrm{T} 5 \mathrm{~g}$ ) and

ii. $\mathrm{A} 3, \mathrm{~T} 2, \mathrm{~T} 7$ and $\mathrm{T} 6 \mathrm{~g}$.

We will prove that $\mathrm{A} 3, \mathrm{~T} 7$ and $\mathrm{T} 4 \mathrm{~g}$ is an axiomatization of $\mathrm{T}_{\mathrm{c}}^{\mathrm{o}}$ (given $\mathrm{T}_{+}^{\mathrm{o}}$, of course). The other two cases can be proved similarly. First, we remark that the following lemma is provable

Lemma 1. The replacement theorem holds for $\mathrm{T}_{\mathrm{c}}^{\mathrm{o}}$. That is, if $\vdash A \leftrightarrow B$ (i.e., if $\vdash A \rightarrow B$ and $\vdash B \rightarrow A$ ), then $A$ and $B$ are interchangeable in any wff in which one of them appears.

Proof. The resources of Routley's basic positive logic $\mathrm{B}_{+}$(see [2]) suffice for the negationless part of $\mathrm{T}_{\mathrm{c}}^{\mathrm{o}}$. Then, use $\mathrm{T} 2$ for the negation case.

This is the proof that $\mathrm{A} 3, \mathrm{~T} 7$ and $\mathrm{T} 4 \mathrm{~g}$ axiomatize $\mathrm{T}_{\mathrm{c}}^{\mathrm{o}}$ :

Proof. Let T4g be T4 $(n=k)$, i.e.,

$$
\begin{aligned}
(1) . & A \rightarrow\left\{[ A \rightarrow [ B _ { 1 } \rightarrow ( ( \ldots ( B _ { k } \rightarrow \neg C ) \ldots ) ) ] ] \rightarrow \left[B_{1} \rightarrow\right.\right. \\
& \left.\left.\left(\left(\ldots\left(B_{k} \rightarrow \neg C\right) \ldots\right)\right)\right]\right\}
\end{aligned}
$$

Next, $\neg C$ is substituted by $\neg\left(B_{k} \rightarrow B_{k}\right)$ in (1):

$$
\begin{aligned}
(2) . & A \rightarrow\left\{[ A \rightarrow [ B _ { 1 } \rightarrow ( ( \ldots ( B _ { k } \rightarrow \neg ( B _ { k } \rightarrow B _ { k } ) ) \ldots ) ) ] ] \rightarrow \left[B_{1} \rightarrow\right.\right. \\
& \left.\left.\left(\left(\ldots\left(B_{k} \rightarrow \neg\left(B_{k} \rightarrow B_{k}\right)\right) \ldots\right)\right)\right]\right\}
\end{aligned}
$$

By $\mathrm{T} 7$ and $\mathrm{T} 8$

$$
\text { (3). } \neg B_{k} \leftrightarrow\left[B_{k} \rightarrow \neg\left(B_{k} \rightarrow B_{k}\right)\right]
$$

By Lemma 1, (2) and (3)

$$
\begin{aligned}
\text { (4). } & A \rightarrow\left\{[ A \rightarrow [ B _ { 1 } \rightarrow ( ( \ldots ( B _ { k - 1 } \rightarrow \neg B _ { k } ) \ldots ) ) ] ] \rightarrow \left[B_{1} \rightarrow\right.\right. \\
& \left.\left.\left(\left(\ldots\left(B_{k-1} \rightarrow \neg B_{k}\right) \ldots\right)\right)\right]\right\}
\end{aligned}
$$

Now, (4) is $\operatorname{T} 4 \mathrm{~g}(n=k-1)$ and it is clear that the procedure can be iterated. Therefore, $\mathrm{A} 3, \mathrm{~T} 4 \mathrm{~g}$ and $\mathrm{T} 7$ do axiomatize $\mathrm{T}_{\mathrm{c}}^{\mathrm{o}}$ (note that $\mathrm{T} 8$ is immediate from $\mathrm{A} 3$ and the identity axiom $A \rightarrow A$ of $\left.\mathrm{T}_{+}^{\mathrm{o}}\right)$. 


\section{Semantics for $T_{c}^{o}$}

A $\mathrm{TW}_{+}$model (see, e.g., [2]) is a quadruple $\langle K, O, R, \models\rangle$, where $K$ is a nonempty set, $O$ a subset of $K$ and $R$ a ternary relation defined on $K$ subject to the following definitions and postulates for all $a, b, c, d \in K$ :

d1. $a \leq b==_{\mathrm{df}}(\exists x \in O) R x a b$

d2. $R^{2} a b c d==_{\mathrm{df}}(\exists x \in K)[R a b x \& R x c d]$

p1. $a \leq a$

p2. $(a \leq b \& R b c d) \Rightarrow$ Racd

p3. $R^{2} a b c d \Rightarrow(\exists x \in K)[R b c x \& R a x d]$

p4. $R^{2} a b c d \Rightarrow(\exists x \in K)[R a c x \& R b x d]$

$\vDash$ is a valuation relation from $K$ to the sentences of the positive language satisfying the following conditions for all propositional variables $p$, wffs $A$, $B$ and $a, b, c \in K$ :

(i) $(a \vDash p \& a \leq b) \Rightarrow b \vDash p$

(ii) $a \vDash A \vee B$ iff $a \vDash A$ or $a \vDash B$

(iii) $a \vDash A \wedge B$ iff $a \vDash A$ and $a \vDash B$

(iv) $a \vDash A \rightarrow B$ iff for all $b, c \in K(\operatorname{Rabc} \& b \vDash A) \Rightarrow c \vDash B$

A formula is valid $\left(\vDash_{T W_{+}} A\right)$ iff $a \vDash A$ for all $a \in O$ in all models.

$\mathrm{A} \mathrm{T}_{+}^{o}$ model (see [7]) is just like a $\mathrm{TW}_{+}$model but with the addition of the postulate

P1. $R^{2} a b c d \Rightarrow R^{3} a b b c d$

$\left(R^{3} a b c d e={ }_{\mathrm{df}}(\exists x, y \in K)(R a b x \& R x c y \& R y d e)\right)$

Then, a $\mathrm{T}_{\mathrm{c}}^{\mathrm{o}}$ model is a quintuple $\langle O, K, S, R, \models\rangle$, where $\langle O, K, R, \models\rangle$ is a $\mathrm{T}_{+}^{\mathrm{o}}$ model and $S$ a subset of $K$ such that $S \cap O \neq \emptyset$. The following clause and the two following postulates are also added:

$(v \neg) . a \vDash \neg A$ iff for all $b, c \in K,(R a b c \& c \in S) \Rightarrow b \not \models A$

PA2. $\left(R^{2} a b c d \& d \in S\right) \Rightarrow(\exists x \in S) R^{2} a c b x$

PA3. $\left(R^{2} a b c d \& d \in S\right) \Rightarrow(\exists x \in S) R^{2} b c a x$

Definition 3. $\vDash A$ ( $A$ is $\mathrm{T}_{+}^{\mathrm{o}}$ valid) iff $a \vDash A$ for all $a \in O$ in all models. 
So, a first and general difference between $\mathrm{T}_{+}^{\mathrm{o}}$ models and $\mathrm{T}_{\mathrm{c}}^{\mathrm{o}}$ models is the selection of a second subset $S$ of the set $K$. As it is known, $O$ is intuitively the set of all prime regular theories (see $\S 4$ below). Now, $S$ would be the set of all prime consistent theories, an inconsistent theory being that containing the negation of a theorem. The condition $S \cap O \neq \emptyset$ guarantees the existence of at least a regular and consistent theory. On the other hand and concerning clause $(v \neg)$, as it was already remarked in the introduction, we conceive this negation as equivalent to the implication of a falsity constant $\perp\left(\neg A==_{\mathrm{df}} A \rightarrow \perp\right)$. Therefore, this constant $\perp$ can be explained by simply stating that theories containing $\perp$ are inconsistent, do not belong to $S$. Then, clause $(v \neg)$ can be elucidated as follows. It is known that in ternary relational semantics conditional formulas are semantically understood according to the clause

$$
(v \rightarrow) . a \vDash A \rightarrow B \text { iff for all } b, c \in K,(R a b c \& b \vDash A) \Rightarrow c \vDash B
$$

So, the clause for $\neg A(A \rightarrow \perp)$ is

$$
(v \neg \prime) . a \vDash A \rightarrow \perp \text { iff for all } b, c \in K,(\operatorname{Rabc} \& b \vDash A) \Rightarrow c \vDash \perp
$$

Now, as remarked above, let a point $a$ be in $S$ iff $a \not \models \perp$. It is clear that $(v \neg)$ and $(v \neg \prime)$ are equivalent. Finally, regarding the semantical postulates, PA2 and PA3 are the corresponding postulates for A2 and A3.

Next, we prove semantic consistency (soundness) of $\mathrm{T}_{\mathrm{c}}^{\mathrm{o}}$. First, we note that the two following lemmas are proved like in the positive semantics, in a similar way:

Lemma 2. For any wff $A$ and points $a, b$, in $K,(a \leq b \& b \vDash A) \Rightarrow a \vDash A$.

Lemma 3. For any wff $A, B, \vDash A \rightarrow B$ iff for all $a$ in $K, a \vDash A \Rightarrow a \vDash B$.

Theorem 1 (semantic consistency). If $\vdash A$, then $\vDash A$.

Proof. Given the semantic consistency of $\mathrm{T}_{+}^{o}$ (see, e.g., [7]), in order to prove that all $\mathrm{T}_{\mathrm{c}}^{\mathrm{o}}$ theorems are valid, we just have to prove that $\mathrm{A} 2$ and $\mathrm{A} 3$ are valid. Use PA2 and PA3, respectively.

We finish this section proving that if $B$ is the negation of a theorem, $B$ is not valid, i.e.,

Proposition 3. If $\vdash A$, then $\not \models \neg A$.

Proof. Let $b \in S \cap O$. By $\mathrm{TW}_{+}$semantics, $R x b b$ for some $x \in O$. As $A$ is a theorem, $b \vDash A$. Then, $x \not \models \neg A$ by clause $(v \neg)$. 


\section{Completeness of $\mathbf{T}_{c}^{o}$}

Given the completenes of $\mathrm{TW}_{+}$(see, e.g., [2]), we only have to prove that clause $(v \neg)$ and postulates PA2 and PA3 hold canonically.

We begin by recalling some definitions. A theory is a set of formulas closed under adjunction and provable entailment. A theory $a$ is prime if whenever $A \vee B \in a$, then $A \in a$ or $B \in a$. A theory is regular if it contains all theorems. Finally, a theory is inconsistent iff it contains the negation of a theorem. Now, we define the canonical model. Let $K^{T}$ be the set of all theories and $R^{T}$ be defined on $K$ as follows: for all formulas $A, B$ and theories $a, b, c \in K^{T}, R^{T} a b c$ iff $(A \rightarrow B \in a \& A \in b) \Rightarrow B \in c$. Further, let $K^{C}$ be the set of all prime theories, $O^{C}$ the set of all regular prime theories, $S^{C}$ the set of all prime consistent theories and $R^{C}$ the restriction of $R^{T}$ to $K^{C}$. Finally, let $\vDash^{C}$ be defined as follows: for any wff $A$ and $a \in K^{C}, a \vDash^{C} A$ iff $A \in a$.Then, the canonical model is the structure $\left\langle K^{C}, O^{C}, S^{C}, R^{C}, \vDash^{C}\right\rangle$.

We prove

Proposition 4. Clause $(v \neg)$ holds canonically.

Proof. i. If $\neg A \in a$, then $\left(R^{C} a b c \& c \in S^{C}\right) \Rightarrow A \notin b$ :

Suppose $\neg A \in a, R^{C} a b c, c \in S^{C}$ and, by reductio, $A \in b$. By T7, $A \rightarrow \neg(A \rightarrow A) \in a$. So, $\neg(A \rightarrow A) \in c$ by $R^{C} a b c$ and $A \in b$. But then $c$ would be inconsistent.

ii. If $\neg A \notin a$, then there are $b \in K^{C}, c \in S^{C}$ such that $R^{C} a b c$ and $A \in b$ :

First, let us suppose that $\neg A \notin a$. By defining the theories $x=\{B$ : $\vdash A \rightarrow B\}$ and $y=\{B: C \rightarrow B \in a$ and $C \in x\}$, it is easy to show that $R^{T}$ axy and $A \in x(\vdash A \rightarrow A)$. Next, we prove that $y$ is consistent. If $y$ is not consistent, then $\neg B \in y$ where $B$ is a $\mathrm{T}_{\mathrm{c}}^{\mathrm{o}}$ - theorem. By definitions, $C \rightarrow \neg B \in a, C \in x, \vdash A \rightarrow C$ for some wff $C$. By suffixing $\left(\mathrm{TW}_{+}\right)$, $\vdash(C \rightarrow \neg B) \rightarrow(A \rightarrow \neg C)$. Then, $A \rightarrow \neg B \in a$. Now, $\vdash(A \rightarrow \neg B) \rightarrow \neg A$ by A3 because $B$ is a theorem. In consequence, $\neg A \in a$ which contradicts the hypothesis. Therefore, $y$ is consistent. Finally, $x$ and $y$ are extended to prime theories $b, c$ such that $R^{C} a b c$ and $A \in b$.

Now, let us prove that the postulates are canonically valid.

Proposition 5. PA2 and PA3 hold canonically.

We start by proving that PA2 is canonically valid, i.e.,

$$
R^{C 2} a b c d \& d \in S^{C} \Rightarrow\left(\exists x \in S^{C}\right) R^{C 2} a c b x
$$


It is clear that canonical PA2 is immediately derivable from the following lemma:

Lemma 4. Let $a, b, c \in K^{T}$, $d$ be a consistent member in $K^{T}$ and $R^{T 2} a b c d$. Then, there is some $x \in S^{C}$ such that $R^{T 2} a c b x$.

Proof. Suppose for some $x \in K^{T}, R^{T} a b x, R^{T} x c d, d$ being a consistent member in $K^{T}$. Define the theories $y=\{B: A \rightarrow B \in a$ and $A \in c\}$, $z=\{B: A \rightarrow B \in y$ and $A \in b\}$ such that $R^{T} a c y$ and $R^{T} y b z$. Next, we prove that $z$ is consistent. Suppose it is not. Then, $\neg A \in z$ for some theorem $A$. By definitions, $C \rightarrow(B \rightarrow \neg A) \in a, B \in b, C \in c$ for some wff $B, C$. By T5, $B \rightarrow(C \rightarrow \neg A) \in a$. Then, $C \rightarrow \neg A \in x$ by $R^{T} a b x$ and $B \in b$. Finally, $\neg A \in d$ by $R^{T} x c d$ and $C \in c$. But then $d$ would be inconsistent. Now, $z$ is extended to a prime consistent theory $x$ such that $R^{T} a c y$ and $R^{T} y b x$.

Next, we prove that PA3 is canonically valid, i.e.,

$$
R^{C 2} a b c d \& d \in S^{C} \Rightarrow\left(\exists x \in S^{C}\right) R^{C 2} b c a x
$$

This postulate follows from the lemma below

Lemma 5. Let $a, b, c \in K^{T}$, $d$ be a consistent member in $K^{T}$ and $R^{T 2} a b c d$. Then, there is some $x \in S^{C}$ such that $R^{T 2} b c a x$.

Proof. Similar to that of Lemma 4. Use T4g $(n=1)$.

Now, given the completeness of $\mathrm{T}_{+}^{o}$ (see [7]) and Propositions 4 and 5, we have

Theorem 2 (completeness of $T_{\mathrm{c}}^{\mathrm{o}}$ ). If $\vDash A$, then $\vdash A$.

We finish this section with the following proposition:

Proposition 6. The corresponding postulates for T1-T8 are:

PT1. $R a b c \& c \in S \Rightarrow(\exists x \in S) R b a x$

PT2. $R^{2} a b c d \Rightarrow(\exists x \in K)(R a c x \& R b x d)$

PT3. $R^{2} a b c d \Rightarrow(\exists x \in K)($ Rbcx \& Raxd $)$

PT4. $R^{2} a b c d \& d \in S \Rightarrow(\exists x \in S) R^{2} b a c x$

PT5. $R^{3}$ abcde \& $e \in S \Rightarrow(\exists x \in S) R^{3} a c b d x$

PT6. $R^{3}$ abcde \& $e \in S \Rightarrow(\exists x \in S) R^{3}$ bcadx 
PT7. $R^{2} a b c d \& d \in S \Rightarrow(\exists x \in K)(\exists y \in S)(R c b x \&$ Raxy $)$

PT8. Rabc \& $c \in S \Rightarrow(\exists x \in O)(\exists y \in S) R c x y$

In PT5 and PT6, $R^{3} a b c d e={ }_{\mathrm{df}}(\exists x, y \in K)(R a b x \& R x c y \& R y d e)$.

Proof. We indicate which negation theorem suffices in each case to prove that the corresponding postulate holds canonically. Then, similarly, the proof proceeds as in Lemma 1 and Lemma 2. (1) PT1: T4; (2) PT2: the suffixing axiom of $\mathrm{TW}_{+} ;(3)$ PT3: the prefixing axiom of $\mathrm{TW}_{+} ;$(4) PT4: T4g $(n=1)$; (5) PT5: T5g $(n=2)$; (6) PT6: T6g $(n=2)$; (7) PT7: T5; (8) PT8: A2.

The suffixing (suf) and prefixing (pref) axioms are

$$
(A \rightarrow B) \rightarrow[(B \rightarrow C) \rightarrow(A \rightarrow C)]
$$

and

$$
(B \rightarrow C) \rightarrow[(A \rightarrow B) \rightarrow(A \rightarrow C)]
$$

respectively. The corresponding postulates are PT2 for suf. and PT3 for pref. They suffice for T2 and T3 because given the definition of the constructive negation, T2 (T3) is actually an instance of suf. (pref.).

For instance, let us prove that PT5 holds canonically. It derives from the following lemma:

Lemma 6. Let $a, b, c, d \in K^{T}$, e being a consistent member in $K^{T}$ and $R^{T 3} a b c d e$. Then, there is some $x \in S^{C}$ such that $R^{T 3} a c b d x$.

Proof. Suppose $R^{3} a b c d e$ (i.e., Rabu, Rucw, Rwde for some $u, w \in K^{T}$ ) and $e \in S$. Define the theories $x, y, z$ such that Racx, Rxby and Rydz. We have to prove that $z$ is consistent. Suppose it is not. Then, $D \rightarrow$ $[C \rightarrow(B \rightarrow \neg A)] \in a, B \in d, C \in b, D \in c$ for some wff $C, B, D$ and theorem $A$. By T5g $(n=2), C \rightarrow[D \rightarrow(B \rightarrow \neg A)] \in a$. By Rabu, $D \rightarrow$ $(B \rightarrow \neg A) \in u$. By Rucw, $B \rightarrow \neg A \in w$. By Rwde, $\neg A \in e$. But then $e$ would be inconsistent.

To finish this section we remark that jointly taken, Propositions 1, 2 and 6 provide a number of equivalent (and corresponding to each other) syntactical and semantic axiomatizations of $\mathrm{T}_{\mathrm{c}}$. But we will not pursue this topic here any further. 


\section{The $\operatorname{logics} \mathrm{E}_{+}^{\mathrm{o}}, \mathbf{R}_{+}^{\mathrm{o}}, \mathrm{RMO}_{+}^{\mathrm{o}}, \mathrm{S} 4_{+}^{\mathrm{o}}, \mathrm{I}_{+}^{\mathrm{o}}$ and $\mathrm{LC}_{+}^{\mathrm{o}}$}

Consider the following axioms and rules of inference:

A4. $A \rightarrow[[A \rightarrow(B \rightarrow C)] \rightarrow(B \rightarrow C)]$

A5. $A \rightarrow(A \rightarrow A)$

A6. $A \rightarrow(B \rightarrow A)$

A7. $(A \rightarrow B) \vee(B \rightarrow A)$

CAP assertion (CAP asser.): If $\vdash A$, then $\vdash[A \rightarrow(B \rightarrow C)] \rightarrow(B \rightarrow C)$ $K$ rule $(K)$ : If $\vdash A$, then $\vdash B \rightarrow A$

The positive logics are:

$\mathrm{E}_{+}^{o}$ : $\quad \mathrm{T}_{+}^{\mathrm{o}}$ plus CAP asser.

$\mathrm{R}_{+}^{\circ}$ : $\quad \mathrm{T}_{+}^{\mathrm{o}}$ plus A4

$\mathrm{RMO}_{+}^{\circ}: \mathrm{R}_{+}^{\mathrm{o}}$ plus $\mathrm{A} 5$

$\mathrm{S} 4_{+}^{\mathrm{o}}$ : $\quad \mathrm{E}_{+}^{\mathrm{o}}$ plus $K$

I+ $\quad \mathrm{T}_{+}^{\mathrm{o}}$ plus $\mathrm{A} 6$

$\mathrm{LC}_{+}^{o}: \quad \mathrm{I}_{+}^{\mathrm{o}}$ plus A7

If in all foregoing formulations we change (whenever present) A1, CAP asser. and A4 for (respectively) contraction

$$
[A \rightarrow(A \rightarrow B)] \rightarrow(A \rightarrow B)
$$

the assertion rule

$$
\text { if } \vdash A \text {, then } \vdash(A \rightarrow B) \rightarrow B
$$

and assertion

$$
A \rightarrow[(A \rightarrow B) \rightarrow B]
$$

we get formulations of the positive logics $\mathrm{T}_{+}, \mathrm{E}_{+}, \mathrm{R}_{+}, \mathrm{RMO}_{+}, \mathrm{S} 4_{+}, \mathrm{L}_{+}$and $\mathrm{LC}_{+}$, respectively. So, $\mathrm{E}_{+}^{\mathrm{o}}, \mathrm{R}_{+}^{\mathrm{o}}, \mathrm{RMO}_{+}^{\mathrm{o}}, \mathrm{S}_{+}^{\mathrm{o}}, \mathrm{I}_{+}^{\mathrm{o}}$ and $\mathrm{LC}_{+}^{\mathrm{o}}$ are restrictions with the CAP of the precedently mentioned logics (see [1], [7]).

The deductive relations these logics maintain with each other (which are exactly those maintained by their unrestricted counterparts) are summarized in the following diagram where the arrow stands for set inclusion: 


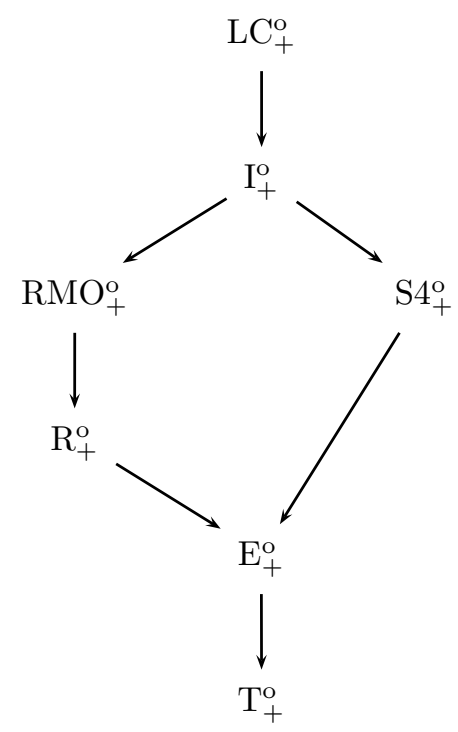

Now, we define the semantics. Consider the following postulates:

PCAPasser. $R a b c \Rightarrow(\exists x \in O) R^{2} a x b c$

PA4. $R^{2} a b c d \Rightarrow R^{2} b a c d$

PA5. $R a b c \Rightarrow a \leq c$ or $b \leq c$

PK. $(\exists x \in O) x \leq a$

PA6. $R a b c \Rightarrow a \leq c$

PA7. (Rabc \& Rade) $\Rightarrow b \leq e$ or $d \leq c$

Then $\mathrm{E}_{+}^{\mathrm{o}}$ models, $\mathrm{R}_{+}^{\mathrm{o}}$ models, $\mathrm{RMO}_{+}^{\mathrm{o}}$ models, $\mathrm{S} 4_{+}^{\mathrm{o}}$ models, $\mathrm{I}_{+}^{\mathrm{o}}$ models and $\mathrm{LC}_{+}^{\mathrm{o}}$ models are defined by adding to $\mathrm{T}_{+}^{\mathrm{o}}$ models PCAPasser., PA4, PA5, $\mathrm{P} K$, PA6, PA7, respectively. A formula $A$ is valid iff $a \vDash A$ for all $a \in O$ in all models. It is easy to prove along the lines of [7] that (for each one of these logics) $A$ is valid iff $A$ is a theorem.

\section{The $\operatorname{logics} \mathrm{E}_{\mathrm{c}}^{\mathrm{o}}, \mathrm{R}_{\mathrm{c}}^{\mathrm{o}}, \mathrm{RMO}_{\mathrm{c}}^{\mathrm{o}}, \mathrm{S} 4_{\mathrm{c}}^{\mathrm{o}}, \mathrm{I}_{\mathrm{c}}^{\mathrm{o}}$ and $\mathrm{LC}_{\mathrm{c}}^{\mathrm{o}}$}

We add the constructive negation of $\S 1$ to each one of the positive logics in the preceding paragraph. In this way, we define the logics $\mathrm{E}_{\mathrm{c}}^{\mathrm{o}}, \mathrm{R}_{\mathrm{c}}^{\mathrm{o}}, \mathrm{RMO}_{\mathrm{c}}^{\mathrm{o}}$, $\mathrm{S} 4_{\mathrm{c}}^{\mathrm{o}}, \mathrm{I}_{\mathrm{c}}^{\mathrm{o}}$ and $\mathrm{LC}_{\mathrm{c}}^{\mathrm{o}}$. Finally, we define the semantics. $\mathrm{E}_{\mathrm{c}}^{\mathrm{o}}$ models, $\mathrm{R}_{\mathrm{c}}^{\mathrm{o}}$ models, $\mathrm{RMO}_{\mathrm{c}}^{\mathrm{o}}$ models, $\mathrm{S} 4_{\mathrm{c}}^{\mathrm{o}}$ models, $\mathrm{I}_{\mathrm{c}}^{\mathrm{o}}$ and $\mathrm{LC}_{\mathrm{c}}^{\mathrm{o}}$ models are just like $\mathrm{T}_{\mathrm{c}}^{\mathrm{o}}$ models save for the addition of the postulates PCAPasser., PA4, PA5, PK, PA6 and PA7, respectively. 
It is obvious that completeness immediately follows from the completeness of $\mathrm{T}_{\mathrm{c}}^{\mathrm{o}}$ and that of the positive logics.

We finish this paragraph with a note. In order to prove the canonical adequacy of the postulates $\mathrm{P} K, \mathrm{PA} 6$ and PA7, the theories in the canonical model must be non-null. Now, let $L$ be any logic with the $K$ rule. Then, it is easy to prove that if a $L$-theory is non-null, then it is regular. Therefore, the theories in the canonical models for $\mathrm{S} 4_{\mathrm{c}}^{\mathrm{O}}, \mathrm{I}_{\mathrm{c}}^{\mathrm{o}}$ and $\mathrm{LC}_{\mathrm{c}}^{\mathrm{o}}$ are regular, whence the sets $K^{C}$ and $O^{C}$ are equivalent. So, it would have been more simple to define $\mathrm{S} 4_{\mathrm{c}}^{\mathrm{o}}\left(\mathrm{I}_{\mathrm{c}}^{\mathrm{o}}, \mathrm{LC}_{\mathrm{c}}^{\mathrm{o}}\right)$ models dispensing with the set $O$ and, accordingly, defining validity in respect of the set $K$. We have disregarded this possibility to keep the exposition general (it is easy to adapt the arguments in this paper to the case of non-null theories. See,.e.g., [11] or [12]).

\section{Proof that all the logics defined have the CAP}

Consider the following set of matrices where the only designated value is 2 :

\begin{tabular}{l|lll|l}
$\rightarrow$ & 0 & 1 & 2 & $\neg$ \\
\hline 0 & 2 & 0 & 2 & 2 \\
1 & 2 & 2 & 2 & 2 \\
2 & 0 & 0 & 2 & 2
\end{tabular}

\begin{tabular}{l|lll}
$\wedge$ & 0 & 1 & 2 \\
\hline 0 & 0 & 1 & 0 \\
1 & 1 & 1 & 1 \\
2 & 0 & 1 & 2
\end{tabular}

\begin{tabular}{l|lll}
$\vee$ & 0 & 1 & 2 \\
\hline 0 & 0 & 0 & 2 \\
1 & 0 & 1 & 2 \\
2 & 2 & 2 & 2
\end{tabular}

This set verifies $\mathrm{LC}_{+}^{\circ}$. Now let $(A \rightarrow B) \rightarrow C$ be any wff in which neither $\rightarrow$ nor $\neg$ appears in $C$. Assign all the variables in $C$ the value 1 . Then, the value of $(A \rightarrow B) \rightarrow C$ is 0 . Consequently, $\mathrm{LC}_{+}^{\circ}$ (and any logic included in it) has the CAP.

\section{References}

[1] Anderson, A. R., and N. D. Jr. Belnap, Entailment. The Logic of Relevance and Necessity, vol. I. Princeton University Press, 1975.

[2] Anderson, A. R., N. D. Belnap Jr., and J. M. Dunn, Entailment. The Logic of Relevance and Necessity, vol. II. Princeton University Press, 1992.

[3] Johansson, I., "Der Minimal Kalkül, ein reduzierte intuitionistischer Formalismus", Compositio Mathematica, 119-136, 1936.

[4] Kamide, N., "A canonical model construction for substructural logics with strong negation", Reports on Mathematical Logic, 36, 95-116, 2002.

[5] Kolmogorov, A.N., "On the principle of tertium non datur". In: Van Heijenoort, From Hegel to Gödel, C.U.P., pp. 414-437, 1967. 
[6] Kowalski, T., and H. Ono, Residuated Lattices: an algebaric glimpse at logics without contraction (preliminary report). JAIST, 2000.

[7] Méndez, J. M., "A Routley-Meyer semantics for converse Ackermann Property", Journal of Philosophical Logic, 16, 65-76, 1987.

[8] Méndez, J.M., "Converse Ackermann Property and semiclassical negation", Studia Logica, 47, 159-168, 1988.

[9] Ono, H., and Y. Komori, "Logics without the contraction rule", Journal of Symbolic Logic, 50, 169-201, 1985.

[10] Robles, G., and J. M. Méndez, "The logic B and the reductio axioms", Bulletin of the Section of Logic, 33, 87-94, 2004.

[11] Robles, G., J. M. Méndez, F. Salto, and P. Méndez R., "Intuitionistic Propositional Logic with the Converse Ackermann Property", Teorema, 22, 43-54, 2003.

[12] Salto, F., J. M. Méndez, and G. Robles, "Restricting the contraction axiom in Dummett's LC: a sublogic of LC with the Converse Ackermann Property, the logic LC ", Bulletin of the Section of Logic, 30, 139-146, 2001.

Gemma Robles

José M. MÉNDEZ

Department of Philosophy and Logic and Philosophy of Science

Universidad de Salamanca

Edificio F.E.S., Campus Unamuno

E-37007, Salamanca, Spain

http://web.usal.es/ sefus

\{gemm, sefus\}@usal.es 